\title{
CORRESPONDENCE
}

\section{Significance of sputum purulence to guide antibiotic therapy in exacerbations of COPD}

\section{To the Editor:}

We read with interest the study by SOLER et al. [1] evaluating role of sputum purulence as a guide for antibiotic use in hospitalised patients with exacerbations of chronic obstructive pulmonary disease (COPD).

Besides bacteria, viruses and air pollution can also lead to exacerbations of COPD. These episodes are characterised by recruitment of leukocytes (neutrophils, eosinophils, etc.), which leads to sputum purulence. The sputum colour/purulence is strongly correlated with markers of bronchial inflammation, like myeloperoxidase (green coloured) and leukocyte elastase present within these inflammatory cells [2], and may not mandate the bacterial presence. Moreover, isolation of bacteria from sputum may represent bronchial colonisation, especially in conditions with co-existent architectural distortion like bronchiectasis.

In the study by SOLER et al. [1], sputum purulence was used as a criterion to initiate antibiotics. It would have been more representative if serial sputum examinations (for purulence) were used to decide the duration of antibiotics as well, thus correlating sputum purulence with total antibiotic exposure. In addition, excluding sick and recently treated patients from the study might have decreased the predictive value of sputum purulence as these patients are more likely to suffer from bacterial infections.

Absence of sputum purulence cannot rule out bacterial infection reliably [3]. In fact, comprehensive evaluation of presumptive signs and symptoms (fever, increased breathlessness and increase in sputum volume/purulence) along with relevant haematological investigations and supported by a physician's decision to treat are the major parameters guiding the use of antibiotics. Instead of conducting randomised control trials on sputum purulence, it may be useful if studies are conducted to evaluate new biomarkers of bacterial infectivity with better sensitivity and specificity and correlate them with crude indicators like sputum purulence.

\section{Deepak Aggarwal*, Prasanta R. Mohapatra* and Phiza Aggarwal ${ }^{\#}$}

*Dept of Pulmonary Medicine, Government Medical College and Hospital, Sec-32, Chandigarh, and ${ }^{\#} \mathrm{MM}$ Institute of Medical Sciences and Research, Mullana, Ambala, India.

Correspondence: D. Aggarwal, Dept of Pulmonary Medicine, Block-D, Level-5, Government Medical College and Hospital, Sec-32, Chandigarh, Pin 160030, India. E-mail: drdeepak@ hotmail.com

\author{
Statement of Interest: None declared.
}

\section{REFERENCES}

1 Soler N, Esperatti M, Ewig S, et al. Sputum purulence-guided antibiotic use in hospitalised patient with exacerbations of COPD. Eur Respir J 2012; 40: 1344-1535.

2 Stockley RA, Bayley D, Hill SL, et al. Assessment of airway neutrophils by sputum colour: correlation with airways inflammation. Thorax 2001; 56: 366-372.

3 David-Ona DA, Dacuycuy MAC, Alejandria MM. The clinical utility of sputum color in relation to bacterial infection among patients with COPD in acute exacerbation. Phil J Microbiol Infect Dis 2001; 30: $120-125$.

DOI: $10.1183 / 09031936.00083112$

From the authors:

We thank D. Aggarwal and co-workers for their interest in our recently published paper regarding a sputum purulence-guided strategy of antibiotic treatment in patients with exacerbations of chronic obstructive pulmonary disease (COPD) requiring hospitalisation [1]. Their comments highlight important issues relating to the discrepancies reported in the literature about the relationship between sputum colour and the presence of pathogenic bacteria in the lower respiratory tract.

First, the question of whether isolation of bacteria from the sputum of patients with COPD is or is not directly related to exacerbations is still a subject of controversy. Classically, the Anthonisen criteria (i.e. increased dyspnoea, sputum production and sputum purulence) have been considered the most reliable predictors of bacterial infection as a cause of exacerbation [2]. Moreover, the most relevant advance in our ability to predict bacterial involvement in COPD exacerbations was the observation that not all three criteria have the same predictive value. STOCKLEY et al. [3], with the aid of colour chart, demonstrated that a change in sputum colour during exacerbations was a sensitive and specific marker for bacterial presence and, more importantly, proved the high accuracy of using mucoid sputum to rule out bacterial infection. These findings have been validated using more specific and sensitive sampling methods such as the protected specimen brush (PSB) in patients with severe COPD exacerbations. In a previously published bronchoscopic study, we found a high concordance between bacterial pathogens in samples retrieved by PSB and sputum sampling [4]. However, it is not feasible to use invasive diagnostic methods in clinical practice, and sputum sampling is still 